ENCYCLOPEDDE Encyclopédie berbère

BERBERE

$2 \mid 1985$

2 | Ad - Ağuh-n-Tahlé

Afrag / Afarag

(clôture, haie, enclos, jardin)

S. Chaker, E. Bernus et G. Camps

\title{
OpenEdition
}

Journals

Édition électronique

URL : http://journals.openedition.org/encyclopedieberbere/885

DOI : $10.4000 /$ encyclopedieberbere.885

ISSN : 2262-7197

Éditeur

Peeters Publishers

Édition imprimée

Date de publication : 1 novembre 1985

Pagination : 206-208

ISBN : 2-85744-209-2

ISSN : $1015-7344$

Référence électronique

S. Chaker, E. Bernus et G. Camps, «Afrag / Afarag », Encyclopédie berbère [En ligne], 2 | 1985, document A77, mis en ligne le 01 décembre 2012, consulté le 25 septembre 2020. URL : http:// journals.openedition.org/encyclopedieberbere/885 ; DOI : https://doi.org/10.4000/ encyclopedieberbere.885

Ce document a été généré automatiquement le 25 septembre 2020

(c) Tous droits réservés 


\section{Afrag / Afarag}

(clôture, haie, enclos, jardin)

\section{S. Chaker, E. Bernus et G. Camps}

1 Nom d'action verbale issu de la racine pan-berbère frg dont la signification la plus générale est : « clôturer, enclore, entourer d'une haie... ».

Localement, en deux points au moins, frq est lié à l'idée de " pouvoir » :

- Touareg méridional : efreg: " pouvoir, être capable».

- Ghadamès : efreg: « l'emporter sur quelqu'un ».

2 Il s'agit probablement d'évolutions sémantiques locales (" enclore $\rightarrow$ séparer $\rightarrow$ tenir à distance $\rightarrow$ être de la force de...»), à moins qu'il n'y ait eu confusion de deux racines initialement distinctes.

3 Ce verbe appartient au fond lexical chamito-sémitique (cf. sémitique frq: «séparer, diviser...» et autres formes voisines) et vraisemblablement à toute l'aire méditerranéenne (cf. parc, park « enclos » de certaines langues indoeuropéennes et dont l'origine est souvent considérée comme obscure).

4 Mais il ne peut en aucune façon s'agir d'un emprunt au sémitique -même ancien- car cette forme est parfaitement intégrée au plan grammatical (notamment dérivationnel) et est attestée dans l'ensemble du monde berbère (totalité du Maroc, Kabylie, Aurès, Touareg Ahaggar...).

5 En tant que nom d'action verbale, afrag/afarag a, en base, la valeur abstraite de : « fait/ action de clôturer ». Partout il glisse, comme beaucoup de déverbatifs abstraits, vers la dénomination d'une réalité concrète : «clôture, haie » et, par métonymie, (contenant $\rightarrow$ contenu) : « enclos, jardin ».

- Maroc : afrag : « haie (sèche de buissons épineux)».

- Kabylie : afrag : « clôture, haie »

-Touareg : afarag: « clôture, haie, enclos, jardin ». (le pluriel fondamental est partout : ifergan).

6 Le sens touareg de «jardin » est explicitement présenté comme récent et secondaire par Ch. de Foucauld (1/343). 
7 Au Maroc, afrag prend aussi le sens de «clôture de toile qui entoure les tentes particulières du sultan en déplacement » (Laoust, 1922, p. 3). Cette signification passée en arabe dialectal marocain semble attestée dès le Moyen Âge où afrag paraît avoir désigné « le camp, la tente royale » (Ibn Khaldoun, IV, p. 201).

En Kabylie, afrag a souvent le sens de «cour, maisonnée, unité domestique (délimitée par la même haie ou le même muret), unité d'habitation, famille élargie ». On dira alors facilement : yiwen wefrag-nney

un clôture-notre = «notre clôture/cour est une » - « nous appartenons à la même unité domestique/à la même famille ».

Dans cet usage, afrag est fortement concurrencé par l'emprunt arabe lhara, « cour ».

La forme connaît les variations phonétiques habituelles déterminées par les tendances de chaque parler; au Maroc on rencontre à côté de la forme de base afrag : ifrig, afraž, afray...

\section{Afarag (pl. Ifergan) (touareg) (E. Bernus)}

9 Ce mot a deux sens chez les Touaregs :

1. clôture, haie.

2. jardin, champ irrigué.

10 1. Afarag désigne d'abord la clôture, la haie. Dans les zones pastorales sans agriculture, c'est une haie faite d'abattis, dont les branches munies d'épines forment des chevaux de frise naturels. Tous les acacias, tamat (Acacia flava), afagag (Acacia raddiana), tiggart (Acacia nilotica), tazzeyt (Acacia laeta), orof (Acacia seyal) et également d'autres arbres épineux tels tiboraq (Balanites aegyptiaca) ou tabakat (Ziziphus mauritiana) servent à construire les clôtures).

11 Dans les campements, des haies entourent les enclos à chèvres et à moutons, à cabris et agneaux, et parfois à vaches. Des haies, dans certains cas, cernent les tentes pour les isoler du bétail.

12 Chez les Iwllemmeden, Afarag est le nom d'une bataille qui opposa en 1865 (Nicolas, 1950, p. 62 et Bernus, 1970, p. 446-447) les Kel-Dinnik et les Kel-Ataram ces derniers, retranchés près de Menaka, construisirent une clôture faite de piquets de bois attachés par des lacets de cuir. Les Kel-Dinnik venus les attaquer avec l'aide des Kel-Aïr et des Kel-Gress réussirent à forcer l'enceinte ce qui donna son nom à la bataille.

13 Les iklan (captifs) sont parfois appelés Deffar-afarag ("derrière la haie »), car dans les campements, leurs tentes sont installées au delà des enclos à bétail. Dans l'arrondissement de Téra (ouest du Niger) une tribu porte ce nom, transcrit par l'administration en Dufarafarak. Cette tribu qui comporte quelques imajeyen et une masse d'iklan a pris le nom de ces derniers, alors que les imajeyen étaient connus sous le nom de Kel-Ansongo.

14 2. Dans les régions d'agriculture irriguée, afarag désigne le jardin et par conséquent le contenant comme le contenu.

Chez les Iwllemmeden Kel-Ataram, les végétaux irrigués portent le nom d'išikraš (cf. Foucauld, 1951-1952, t. I, p. 343 azekrih).

Les haies de ces jardins sont également faites de branchages d'épineux, soutenus souvent de troncs de tirza (Calotropis procera) qui encadrent parfois des portes d'accès. 

les enclos provoque une déforestation grave tout autour des zones cultivées. A In Gall, où les arbres les plus proches se trouvent dans des vallées distantes de 6 ou $7 \mathrm{~km}$, les habitants doivent, pour enclore les parcelles de jeunes dattiers, acheter des charges de branches épineuses apportées sur la tête.

Dans les zones agricoles sud-sahéliennes, la haie, qui parfois entoure le champ de culture pluviale pour le protéger de la divagation des troupeaux, porte aussi le nom d'afarag. Par contre, un tel champ, qui ne reçoit que l'eau des pluies en une courte saison, s'appelle tawgest pl. šiwugas et s'oppose au champ irrigué afarag pl. ifergan.

\section{Afarag (ville) (G. Camps)}

acienne qui, à l'emplacement actuellement occupé par la citadelle sur le mont Acho, dominait le site de Ceuta. Cette localité doit son nom à la même racine «efreg " (entourer d'une clôture).

Cette racine entre dans la composition de nombreux toponymes.

\section{BIBLIOGRAPHIE}

ALOJALY GH. Lexique touareg-français, Copenhague, 1980 (-efreg : « pouvoir, ê. capable », p. 41, -

afarag: « clôture, jardin », p. 41).

BERNUS E. Récits historiques de l'Azawaq. Traditions des Iullemmeden Kel Dinnik. Bull. de l'I.F.A.N., t. XXXII, sér. B, 1970, p. 434-485.

COHEN M. Essai comparatif sur le vocabulaire et la phonétique du chamitosémitique, Paris, 1947 (1969),

(frg: n. ${ }^{\circ} 369$, p. 169).

DALLET J.-M. Dictionnaire kabyle-français, Paris, Selaf, 1982 (efreg, afrag : p. 221).

ERNOUT A. et MEILLET A. Dictionnaire étymologique de la langue latine, 1939 (cf. parco-).

FOUCAULD CH. DE. Dictionnaire touareg-français (4 vol.), 1951-52 (efreg/afarag : I, p. 342).

GRANDSAIGNES D'HAUTERIVES R. Dictionnaire des racines des langues européennes, Paris, 1949 (park : p. $145)$.

IBN KHALDOUN. Histoire des Berbères, Paris (nlle édition), 1925 (4 vol.), (afrag : IV, 201, Index p. 490).

LANFRY J. Ghadamès, II, Alger, 1970, FDB (efreg : p. 96, n. ${ }^{\circ} 0422$ « l'emporter sur quelqu'un ».

LAOUST E. Mots et choses berbères, Paris, 1920 (afrag: p. 3).

LAOUST E. Cours de berbère marocain, Paris, 1921 (ifrig : p. 2 [Souss]).

NICoLAS F. Tamesna. Les Ioullemmeden de l'Est ou Touareg Kel Dinnik. Cercle de Tawa, colonie du Niger, Paris, Imp. nat. 1950.

Encyclopédie berbère, 2 | 1985 
PRASSE K. G. Vocabulaire touareg (Tawellemmet de l'Est-Français), Copenhague, 1970 (efreg : p. 106 : «pouvoir », afarag: p. 11 : « clôture, haie »).

INDEX

Mots-clés : Linguistique, Agriculture, Ville, Touareg 